\title{
News
}

\section{Conference Announcement: Libraries and Information Institutions in the Digital Age (LIDA) 2020}

Theme: Reshaping Identity in The Digital Age: People, Libraries, Data, Technology \& Ethics 19-22 June 2020 Inter-University Centre, Dubrovnik, Croatia (https:// www.iuc.hr/)

Website: http://lida.ffos.hr/

Facebook: https://www.facebook.com/lida2020/

Twitter: @LIDADubrovnik20

E-mail: lida@unizd.hr

Deadline for abstract submission: 1 October 2019

Submissions: https://easychair.org/conferences/?conf=lida2020

\section{About the conference}

Libraries in the Digital Age (LIDA) addresses the changing and challenging environment for libraries and information systems and services in the digital world. LIDA is an international biennial conference that brings together researchers, educators, students, practitioners, and developers from all over the world in a forum for personal exchanges, discussions, and learning, made easier by being held in memorable environs. This year's theme is "Reshaping Identity in The Digital Age: People, Libraries, Data, Technology \& Ethics." We welcome papers that address critical and theoretical examination of the theme; present current research and evidence, as well as examination of best practices from the field, and practitioner perspectives and applications. The year 2020 celebrates the 20th year of LIDA and the return of LIDA to Dubrovnik, Croatia.

\section{Contributions}

Papers, panels, workshops and posters (types described below) are invited covering the following, and related, topics with regard to Libraries, Archives, Museums, and other Information Institutions. Submissions are invited that address the overarching topics: 
- 2020 vision for libraries in the digital age: What is the identity and role of libraries in the digital age?

- Factors shaping and transforming identity in the digital age

- Understanding people's information needs, seeking and use in the context of digital environments and digital services

- Identity, culture, and communication in digital environments

- Identity, community, and belonging - the role of libraries

- Identity, agency and activism, social justice and social inclusion

- Identity, difference and diversity: system and service design

- Digital inclusion and access

- Digital policies, politics and power

- Intellectual freedom and censorship

- Ethics

- Digital environments and the construction and control of identity

- How are identities controlled by the digital environment?

- LIS profession

- Education and training, reference

- Changes and innovation in services, resources and systems

- Digital wellbeing and digital safety

- Protection of digital lives: topics around privacy, digital issues such as cyberbullying, digital harassment, phishing, identity theft and role of libraries

- Heritage, digitization and preservation of resources of and for diverse groups

- Investigating digital networks and network analysis

- Subcultures and marginalized identities

- Innovative approaches to methods, measurement and evaluation: social network analysis, interpretivist methods, altmetrics, data analytics, and visualizations

- Scholarly identity, academic social media sites, roles for libraries

- Thinking ahead out-of-the box: promises and pitfalls of libraries and identity

\section{Types of contributions invited}

1. Papers: up to 20 minute presentations on scholarly research, practical advances, best practices, and educational projects. Both completed research and early work/preliminary results are invited. Submit 1,500 word abstract, plus references by 1 October 2019 .

2. Panels: up to 90 minute sessions that will be interactive and offer different perspectives and approaches to a specific topic. Authors must propose the format and invite up to five panelists (including the moderator). Submit 750 word abstract, plus references by 1 October 2019 .

3. Workshops: up to 90 minute sessions that will be tutorial and educational in nature, and are intended to foster interactive discussions for attendees who share common interest. Submit 750 word abstract, plus references by 1 October 2019. 
4. Posters: short graphic presentations that will be presented in a special Minute Madness session. Awards will be given for Best Poster. Submit 750 word abstract, plus references by 1 October 2019.

5. PhD Forum: short presentations by doctoral students, particularly as related to their dissertation. The PhD Forum provides doctoral students the opportunity to present their work to senior faculty in relatively informal setting and to receive feedback on their dissertation by a panel of international educators. Submit 750 word abstract, plus references by 1 October 2019.

6. Student Showcase: short presentations by undergraduate and graduate students, related to their academic research, practical projects, etc. The showcase will provide students with opportunity to get feedback on their work in informal setting and advice on how to develop their work further and get published. LIDA 2020 Outstanding Student Award will be given for best presentations in this section. Submit 750 word abstract, plus references by 1 October 2019.

All proposal will be refereed in a double-blind process and MUST follow formal LIDA guidelines available at LIDA 2020 website (http://lida.ffos.hr). Proposals will not go forward for review if templates are not used. The conference language is English and all work should be in English, original and not previously presented or published.

Authors can chose to participate in the conference only with the presentation OR both to present their work and submit a full-text manuscript. In both cases, at least one author must be registered and present at the conference.

All authors are invited to submit a full-text manuscript of their paper presented at the LIDA conference to be considered for publication in the peer-reviewed scholarly journal Education for Information (https://www.iospress.nl/journal/educationfor-information/). Education for Information (EFI) welcomes a broad perspective on issues related to the information and communication discipline and is indexed in Cambridge Scientific Abstracts, EBSCO, ERIC, Scopus, Web of Science: Emerging Sources Citation Index, etc. EFI publishes full-length articles (5,000-8,000 words of text excluding the references) and short articles $(1,000-$ 1,500 words of text excluding references), and LIDA authors are invited to submit papers in any of these two categories. Submission guidelines for EFI are available at http://www.iospress.nl/journal/education-for-information/?tab=submissionof-manuscripts. Authors should indicate in their cover letter that their manuscript is a full-text paper of their presentation given at LIDA 2020. Submissions will be subject to a double blind review process and should be submitted via the journal's online system at https://mstracker.com/submit_0.php.

Further queries regarding publication of LIDA 2020 papers in Education for Information can be addressed to Fidelia Ibekwe, EFI editor-in-chief, (fidelia.ibekwesanjuan@univ-amu.fr) and to Marie Radford (mradford@comminfo.rutgers.edu). 


\section{Deadlines}

- Submission of proposals to LIDA 2020 (extended abstracts): 1 October 2019 Notification of acceptance: 15 November 2019

- Submission of full-text manuscripts for consideration for publication in Education for Information (EFI): 30 June 2020 (OPTIONAL)

- Notification of acceptance of full-text manuscripts for publication in EFI: $\mathbf{1}$ October 2020

- Submission of final versions of full-text manuscripts for EFI: 30 October 2020

- Publication of peer reviewed conference papers in EFI: December 2020

\section{Invitation to institutions}

Libraries, information agencies, professional organizations, publishers, and service providers are invited to consider participation at LIDA by providing a demonstration, workshop, or exhibit about their products, services or advances, or by presenting a paper or poster about their activities, as related to the two themes. Sponsorship of an event is also invited. Institutions can benefit as well: we will provide course materials to participants so that they can communicate and transfer topics of interest to their institution.

\section{Conference co-directors}

Drahomira Cupar, Ph.D.

Department of Information Science

University of Zadar, Croatia

dcupar@unizd.hr

Sanjica Faletar Tanackovi? Ph.D.

Department of Information Science

University of Osijek, Croatia

sfaletar@ffos.hr

Ross J. Todd, Ph.D.

School of Communication and Information

Rutgers University, USA

rtodd@rutgers.edu

Marie L. Radford, Ph.D.

School of Communication and Information

Rutgers University, USA

mradford@rutgers.edu 


\section{Venue}

Dubrovnik, Croatia, is recognized as one of the World Cultural Heritage sites by UNESCO. It is a walled city, preserved as it existed in medieval times. A beautiful natural location on the Adriatic Sea, a lavish architecture of squares, palaces, and churches, small, intriguing hill-hugging streets, pedestrian-only traffic within the walls, outings to the enchanting nearby islands - all these and more combine to make Dubrovnik one of the most popular destinations in Europe. The city of Dubrovnik is also often referred to as "the Pearl of the Adriatic." Lately it has become even more popular around the world as a filming location for several famous series and movies: Game of Thrones, Star Wars: The Last Jedi, and The Borgias. 